\title{
Social relationships, knowledge and adjustment to multiple sclerosis
}

\author{
CP MAYBURY,${ }^{*}$ CR BREWIN $\dagger$ \\ From the Department of Psychology, Clifton Hospital, York, ${ }^{*}$ and the MRC Social Psychiatry Unit, Institute \\ of Psychiatry, London, $\dagger U K$
}

SUMMARY A number of factors thought to influence psychological adjustment to multiple sclerosis were studied. Patients' knowledge about their disorder was assessed, but neither their knowledge, level of disability, nor demographic characteristics were related to adjustment. Better adjustment was however associated with having more contact with able-bodied people.

Poor psychological adjustment is common in patients suffering from multiple sclerosis. Surridge ${ }^{1}$ reported that $27 \%$ of his community sample of multiple sclerosis patients were depressed, the symptoms being probably psychogenic in nature. Dalos $e t$ $a l^{2}$ measured the prevalence of emotional disturbance in a sample of clinic attenders, dividing patients into those whose symptoms were in remission, and those with either an acute exacerbation of symptoms or with a progressive, non-remitting form of multiple sclerosis. They found that $90 \%$ of the latter group had some degree of emotional disturbance, while the figure was $39 \%$ for the group whose symptoms were in remission.

Little research has been conducted on the psychological factors which determine the patient's adjustment to multiple sclerosis. Matson and Brooks ${ }^{3}$ have proposed a stage model of adjustment following diagnosis, in which patients progressively pass through the stages of denial, resistance, affirmation and integration. In another descriptive study Miles $^{4}$ has identified two alternative coping strategies used by married couples with one partner suffering from multiple sclerosis. The normalisation strategy involves continuing social relationships according to the pre-illness pattern and avoiding other sufferers. In contrast, the disassociation strategy involves withdrawing from relationships with non-sufferers, sometimes by moving house.

It is not known which, if either, of these two cop-

Address for reprint requests: Mrs CP Maybury, Clifton Hospital, York YO3 6RD, UK.

Received 7 July 1983 and in revised form 28 October 1983. Accepted 5 November 1983 ing strategies identified by Miles has better implications for adjustment. Another possibility is that it is the total amount of social support available, whether from sufferers or non-sufferers, which is important. This prediction follows from Cobb's ${ }^{5}$ finding that social support helps to reduce stress in a wide range of medical and psychiatric disorders. Finally, it is possible that greater knowledge about the disorder leads to better adjustment. The present study attempted to assess the level of knowledge that patients with multiple sclerosis have about their disorder, and to determine which factors are associated with improved adjustment.

\section{Methods}

Thirty-six patients, 24 female and 12 male, from neurologists working in Leeds hospitals and from the Welfare Officer of the Multiple Sclerosis Society (Leeds branch) were studied. Thirty-three of the patients had a definite diagnosis of multiple sclerosis according to the criteria of Rose $e t a l,{ }^{\circ}$ while for the three remaining patients it was the most plausible diagnosis. Seven appeared to be in an acute phase or progressively deteriorating, and 29 patients were in a stable phase of the disorder. No patient refused to take part in the study, although one was found to be too ill to participate. Patients had been aware of their diagnosis for a maximum of seven years (mean 3.7 years). Their average age was 42 years. There were eight people currently employed, six of them full-time. Twenty-four patients were married and living with their spouse; of the remaining twelve, there were three single people, one widow and five divorcees. Level of disability in the sample ranged from minimal neurological signs (Kurtzke Grade I) to quite major impairment (Kurtzke Grade VIII). ${ }^{?}$

Two self-report measures of adjustment were employed. The General Health Questionnaire (GHQ), although originally designed as a screening questionnaire, is often used 
as a measure of minor psychiatric disturbance. All the different versions of the GHQ correlate well with one another, ${ }^{8}$ and the GHQ-28 has been used to detect emotional disturbance which required further investigation in a sample of multiple sclerosis patients.' In the present study, responses to the GHQ-12 were scored on a four-point scale, giving a possibie total score in the range $0-36$. The second measure of adjustment was Rosenberg's $\mathbf{s}^{10}$ selfesteem scale. Once again the items in this ten-item Guttman scale were scored on a four-point scale, giving a total score in the range $0-30$.

A scale measuring normalisation/disassociation was constructed, based on four criteria mentioned by Miles. ${ }^{4}$ These were: (a) whether or not the patients had moved house after multiple sclerosis was diagnosed, irrespective of reason, (b) whether the number of hours per week they spent in social contact with able-bodied people (excluding members of their immediate household) was above or below the mean able-bodied contact in the total sample, (c) whether the number of hours per week of social contact with other disabled people (excluding members of their immediate household) was above or below the mean disabled contact in the total sample, (d) whether or not they were members of the Multiple Sclerosis Society.

Patients were given a score of $\mathbf{0}$ for a response indicating normalisation, that is, not moving house after multiple sclerosis was diagnosed, a high number of contact hours with the able-bodied, a low number of contact hours with the disabled, and non-membership of the Multiple Sclerosis Society. They were given a score of 1 for the opposite (disassociation) response, giving a range for the total scale of $0-4$.

Knowledge of multiple sclerosis was measured by asking patients 14 questions about their disorder, based on information provided in the Multiple Sclerosis Society booklet "So You Have MS?" Patients were assigned a score of 0,1 or 2 on each question depending on the adequacy of their response (details of the scoring criteria are given in the Appendix). All measures were collected during the course of a structured interview, of which all but three were carried out in the patients' own homes.

\section{Results and discussion}

\section{KNOWLEDGE OF THE DISORDER}

Patients' responses to the knowledge questions are presented in the table. A score of nought was assigned to a wrong answer, one to a partial answer, and two to a reasonably complete answer. Individuals' total scores ranged from 7-28 with a mean of 16.7. There was a nonsignificant trend for the 29 Multiple Sclerosis Society members to score more highly than the seven non-members (means $17 \cdot 3$ and 13.9; $\mathrm{t}=1.63, \mathrm{p}<0.12$. Two-tail tests of significance are used throughout). Patients who knew more about their disorder tended to be younger $(\mathrm{r}=-0.45, \mathrm{p}<0.01)$ and more educated $(r=0.36, p<0.05)$. However, within this recently diagnosed group knowledge was unrelated to length of time since diagnosis $(r=-0.09, p>0.10)$ or severity of disability $(r=-0.08, p>0 \cdot 10)$. The table shows that the questions were not all equally well answered. Patients were particularly likely to be unsure about the causes of multiple sclerosis, about what help to expect from their doctor, about what happens during a remission, and about what factors made the symptoms better.

\section{FACTORS ASSOCIATED WITH ADJUSTMENT}

The two measures of self-reported adjustment, GHQ and self-esteem, were strongly correlated ( $\mathrm{r}=$ $0.47, \dot{\mathrm{p}}<0.005)$. Neither measure of adjustment was however found to be correlated with patients' knowledge about their disorder, with their normalisation/disassociation scores, nor with their sex, age, length of time since diagnosis, number of years in full-time education, marital status or level of disability (all ps $>0 \cdot 10$ ).

Next, the relationship of adjustment to the social

Table Knowledge of multiple sclerosis among 36 affected patients

\begin{tabular}{|c|c|c|c|c|}
\hline \multicolumn{2}{|c|}{ Question } & \multicolumn{3}{|c|}{ Response score } \\
\hline & & $\frac{0}{23}$ & $\begin{array}{l}1 \\
3\end{array}$ & $\frac{2}{10}$ \\
\hline $\begin{array}{l}(1) \\
(2) \\
(3) \\
(5) \\
(6) \\
(7) \\
(8) \\
(9) \\
(10) \\
(11) \\
(12) \\
(14)\end{array}$ & $\begin{array}{l}\text { Do you know what causes multiple sclerosis? } \\
\text { What symptoms can you get? } \\
\text { Does everyone get the same symptoms? } \\
\text { How do doctors know that you have multiple sclerosis? } \\
\text { What help can doctors give you? } \\
\text { Is there a cure for multiple sclerosis? } \\
\text { Is multiple sclerosis infectious? } \\
\text { Is multiple sclerosis hereditary? } \\
\text { Give an example of another similar disease of the nerves. } \\
\text { What happens during a relapse? } \\
\text { What happens during a remission? } \\
\text { Are the symptoms of multiple sclerosis the same all the time? } \\
\text { Does anything make the symptoms worse? } \\
\text { Does anything make the symptoms better? }\end{array}$ & $\begin{array}{r}23 \\
5 \\
9 \\
3 \\
18 \\
0 \\
1 \\
5 \\
10 \\
16 \\
26 \\
12 \\
11 \\
21\end{array}$ & $\begin{array}{r}3 \\
12 \\
21 \\
12 \\
- \\
- \\
- \\
-9 \\
\frac{3}{14} \\
13\end{array}$ & $\begin{array}{c}10 \\
19 \\
27^{*} \\
12 \\
6 \\
36^{*} \\
35^{*} \\
31^{*} \\
26^{*} \\
11 \\
7 \\
24^{*} \\
11 \\
2\end{array}$ \\
\hline
\end{tabular}

${ }^{*}$ Responses on these items were given scores of 0 or 2 only. 
contact components of the normalisation/ disassociation score was examined. Although adjustment was not related to level of contact with other disabled people (ps $>0 \cdot 10$ ), patients who had greater contact with the able-bodied had higher self-esteem $(r=-0.44, p<0.01)$ and tended also to have lower GHQ scores $(r=-0.32, p<0.06)$. Patients who had more contact with the able-bodied had other characteristics too: they were significantly less disabled, younger, and more likely to be employed. Partial correlations were therefore calculated to examine the relationships with adjustment when these potential confounding factors were controlled for. It was found that more contact with the ablebodied was still significantly related to self-concept $(r=-0.40, p<0.05)$ and to better psychological health as measured by the GHQ $(r=-0.40, p<$ $0 \cdot 05)$.

In spite of considerable individual variation in knowledge about multiple sclerosis, the present study found no linear relationship between knowledge and psychological adjustment. This may reflect the fact that the relationship is a complex one: some people who are distressed about their condition may deliberately seek more information while others may deliberately avoid it. Alternatively the results may reflect the fact that our current state of knowledge about multiple sclerosis cannot provide much reassurance to the patient. It is also noteworthy that patients were particularly unlikely to know what help to expect from their doctor and what happens during a remission. Improved adjustment may not follow from a "textbook" knowledge of the facts of multiple sclerosis, but may only result from knowledge that can be put to some practical use. This suggests that patient education might usefully focus on practical matters of direct relevance to the patient trying to manage his or her condition.

Like that of Dalos et al, ${ }^{2}$ the present study found that adjustment was unrelated to patients' demographic characteristics or level of disability. Adjustment was also unrelated to the amount of contact with other disabled people. This lack of association must be regarded as tentative because the variation in contact with the disabled (range $0-12$ hours per week) was much less than with the able-bodied (range 0-60 hours per week). Thus it is possible that the restricted range of scores for contact with the disabled reduced the size of the correlation coefficient and contributed to the negative finding. Of particular interest, however, was the positive association between adjustment and contact with the able-bodied. If it can be demonstrated that this contact has some causal influence on adjustment, then we may soon be in a position to make useful recommendations to newly-diagnosed multiple sclerosis patients trying to cope more effectively with their disorder.

We thank Dr S Currie, Dr E Spokes and Dr D Sumner for allowing us to see patients under their $\delta \frac{7}{0}$ care and Mrs I Belton for her help in contacting members of the Multiple Sclerosis Society. We are also grateful to Professor RHS Mindham for comments on an earlier draft of this paper and to Dr $\mathbf{J}$ Howe for help and advice during the study.

\section{Appendix: Scoring for knowledge of multiple sclerosis scale}

(1) What causes multiple sclerosis?

A: Doctors do not know, still researching A virus

Respondent has no idea, or anything else

2 pts
1 pt
0 pts

(2) What symptoms can you get?

A: $\quad$ (i) Blurring of vision and/or double vision

(ii) Slurring of speech

(iii) Weakness of an arm or leg, or clumsiness of a hand

(iv) Altered feelings in arms or legs such as tinglings or numbness ("walking on cotton wool")

(v) Giddiness or lack of balance

(vi) Fatigue which is out of proportion to what one has done, or unpredictable fatigue

(vii) The need to urinate frequently and/or urgently

(3) Does everyone get the same symptoms?

A: $\quad$ No they differ from person to person Yes
4 to 7 of the answers correct-2 pts

2 to 3 of the answers correct-1 pt

0 to 1 of the answers correct-0 pts 
(4) How do doctors know that you have multiple sclerosis?
A:
(i) They look at the history of the illness
Both answers
(ii) They carry out tests, eg. VER, CSF etc.
correct-2 pts
Physical examination, don't know or other
One answer
correct-1 pt
0 pts

(5) What help can doctors give you?
A:
(i) Drugs (steroids) for acute attacks
2 to 4 of the answers
(ii) Contact other professionals, e.g. Social Workers, mentioned-2 pts Physiotherapists etc., who become involved as necessary
(iii) Operations for some tremors etc.
1 of the answers mentioned-1 pt
0 of the answers
mentioned-0 pts

(iv) Help to obtain wheelchairs and other facilities

(6) Is there a cure for multiple sclerosis?
A: No
2 pts
Yes
0 pts

(7) Is multiple sclerosis infectious?
A: No
2 pts
Yes
0 pts

(8) Is multiple sclerosis hereditary?
A: No
2 pts
Yes
0 pts

(9) Give an example of another similar disease of the nerves. If unable, ask if similar to depression?
A: Any neurological condition
Not like depression
2 pts
Like anxiety, depression, or psychiatric disorder
2 pts
0 pts

(10) What happens during a relapse

A: $\quad$ Scarring or hardening (sclerosis) in patches affecting the myelin 2 pts sheath surrounding the nerve, or damage to the nerve sheath

Inflammation of the myelin sheath 2 pts

Damage to nerves $1 \mathrm{pt}$

Anything else 0 pts

(11) What happens during a remission?

A: Recovery, healing of the scar or inflammation of the myelin sheath 2 pts

The nerves heal up

Anything else not indicating healing of the nerves

$1 \mathrm{pt}$

0 pts

(12) Are the symptoms of multiple sclerosis the same all the time?

A: No-they vary from day to day/hour to hour/month to month 2 pts

Yes 0 pts 
(13) Does anything make the symptoms worse?

A: $\quad$ (i) Getting overtired

(ii) Worry and anxiety

(iii) Heat and cold

(iv) A cold or infection, e.g. flu

(13) Does anything make the symptoms better?

A:

(i) Not getting overtired

(ii) Keeping calm/happy/relaxed

(iii) ACTH (steroid) for some

(iv) A special diet helps some
2 to 4 answers

mentioned-2 pts

1 of the answers

mentioned-1 pt

0 of the answers

mentioned-0 pts

2 to 4 of the answers

mentioned-2 pts

1 of the answers

mentioned-1 pt

0 of the answers

mentioned-0 pts

\section{References}

' Surridge D. An investigation into some psychiatric aspects of multiple sclerosis. $\mathrm{Br} J$ Psychiatry 1969;115:749-64.

2 Dalos NP, Rabins PV, Brooks BR, O'Donnell P. Disease activity and emotional state in M.S. Ann Neurol 1983;13:573-7.

${ }^{3}$ Matson RR, Brooks NA. Adjusting to multiple sclerosis: an exploratory study. Soc Sci Med 1977;11:245-50.

${ }^{4}$ Miles A. Some psychosocial consequences of multiple sclerosis: problems of social interaction and group identity. Br J Med Psychol 1979;52:321-31.

${ }^{5}$ Cobb SC. Social support as a moderator of life stress. Psychosom Med 1976;38:300-14.

${ }^{6}$ Rose AS, Ellison GW, Myers LW, Tourtellotte WW. Criteria for the clinical diagnosis of multiple sclerosis. Neurology (Minneap), 1976;26:20-2.

${ }^{7}$ Kurtzke JF. Clinical manifestations of multiple sclerosis. In: Vinken PJ, Bruyn GW, eds. Handbook of Clinical Neurology. Vol. IX. Amsterdam: North-Holland, 1970.

${ }^{8}$ Goldberg DP, Rickels K, Downing R, Hesbacher P. A comparison of two psychiatric screening tests. $\mathrm{Br} \mathrm{J}$ Psychiatry 1976;129:61-7.

${ }^{9}$ Rabins PV, Brooks RR. Emotional disturbance in multiple sclerosis patients: validity of the General Health Questionnaire. Psychol Med 1981;11:425-27.

${ }^{10}$ Rosenberg M. Society and the Adolescent Self-image. Princeton U.P., 1965. 